\title{
Simplifying Observation of Hydrogen Trapping in Atom Probe Tomography
}

\author{
Yi-Sheng Chen ${ }^{1}$, Daniel Haley ${ }^{1}$, Paul A.J. Bagot ${ }^{1}$ and Michael P. Moody ${ }^{1}$ \\ ${ }^{1 .}$ Department of Materials, Oxford University, Oxford, UK
}

The presence of hydrogen within the microstructure of an alloy can lead to a serious reduction of ductility known as hydrogen embrittlement. Although the exact mechanisms of this phenomenon are still subject to debate [1], some mitigation strategies are available. Such strategies include minimizing hydrogen ingress with surface coatings, or controlling hydrogen diffusion within via the introduction of microstructural 'traps', e.g. second phase precipitates. One system of interest in ferritic steels is a microstructure containing finely distributed nano-sized vanadium carbide precipitates $\left(\sim 10 \mathrm{~nm} \mathrm{M}_{4} \mathrm{C}_{3}\right)$, which show good resistance to hydrogen environments [2]. However, to date, there is a lack of experimental techniques to directly study the interaction between hydrogen and microstructural features, limiting researchers' ability to design effective microstructural hydrogen traps.

Atom Probe Tomography (APT) is one experimental technique which may provide insight into hydrogen trapping [3]. Takahashi et al. used a specially modified atom probe to study trapped hydrogen in fine carbides, by charging the sample with deuterium (D) to distinguish loaded hydrogen from background hydrogen [4]. However since then, hydrogen-trapping studies using APT have not advanced significantly, as there exist few systems which can provide an in-situ D loading and subsequent cryotransfer capabilities [3]. A simplified experimental configuration to enable the direct observation of hydrogen via APT is proposed here.

A straightforward electrolytic method to introduce D into APT needle specimens effectively has been demonstrated [5]. This work indicated that ex-situ loading could be potentially followed by a cryotransfer procedure to retain charged D while loading to the APT vacuum chamber. In this work, we demonstrate the realization of such a procedure, where $\mathrm{D}$ charging can be combined with a cold chain to retain deuterium within the sample.

A schematic of the proposed solution is illustrated as Fig.1. After electrochemical D loading (Fig. 1-a), the APT steel sample is rapidly loaded into a thermally-insulated atom probe holder, this is then plunged into liquid nitrogen $\left(\mathrm{LN}_{2}\right)$, attached to a pre-cooled cryo-block, and covered (Fig. 1-b). The entire $\mathrm{LN}_{2}$ unit is then moved into a glove box attached to an atom probe, in order to limit the supply of environmental moisture (Fig. 1-c). After loading into the atom probe, samples can be transferred, whilst still remaining at cryogenic temperatures throughout.

The resultant APT mass spectrum (Fig.2) shows a strong D signal (2 Da) as compared to background hydrogen $(1 \mathrm{Da})$. The result show that the proposed method can retain charged $\mathrm{D}$, in hydrogen-trapping microstructures, with trapping energies greater than or equal to that of the vanadium carbides used here (33-35 $\mathrm{kJ} / \mathrm{mol}[2])$. We believe the proposed configuration, which requires no modification to commercial atom probe designs, can be reasonably reproduced at any other laboratory, making the procedure amenable to industrial research [6]. 
References:

[1] I.M. Robertson, et al., Metall Mater Trans A 46a (2015) p. 2323.

[2] H. Bhadeshia, Isij Int 56 (2016) p. 24.

[3] R.K.W. Marceau, Mater Sci Tech-Lond 32 (2016) p. 209.

[4] J. Takahashi, et al., Scripta Mater 67 (2012) p. 213.

[5] D. Haley, et al., Int J Hydrogen Energ 39 (2014) p. 12221.

[6] The authors acknowledge funding from EPSRC, EP/L014742/1

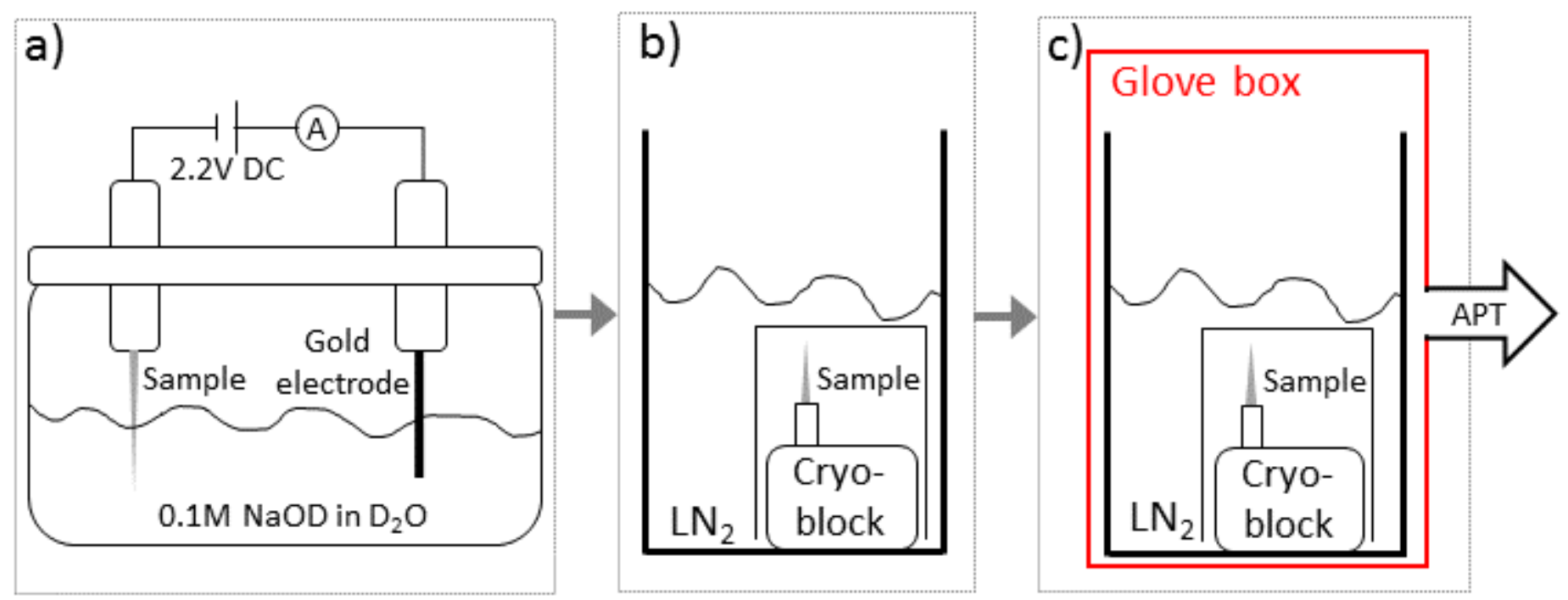

Figure 1. Schematics of the configuration of: (a) electrochemical charging of deuterium; (b) and (c) the subsequently proposed cold-chain for retaining charged D for APT test.

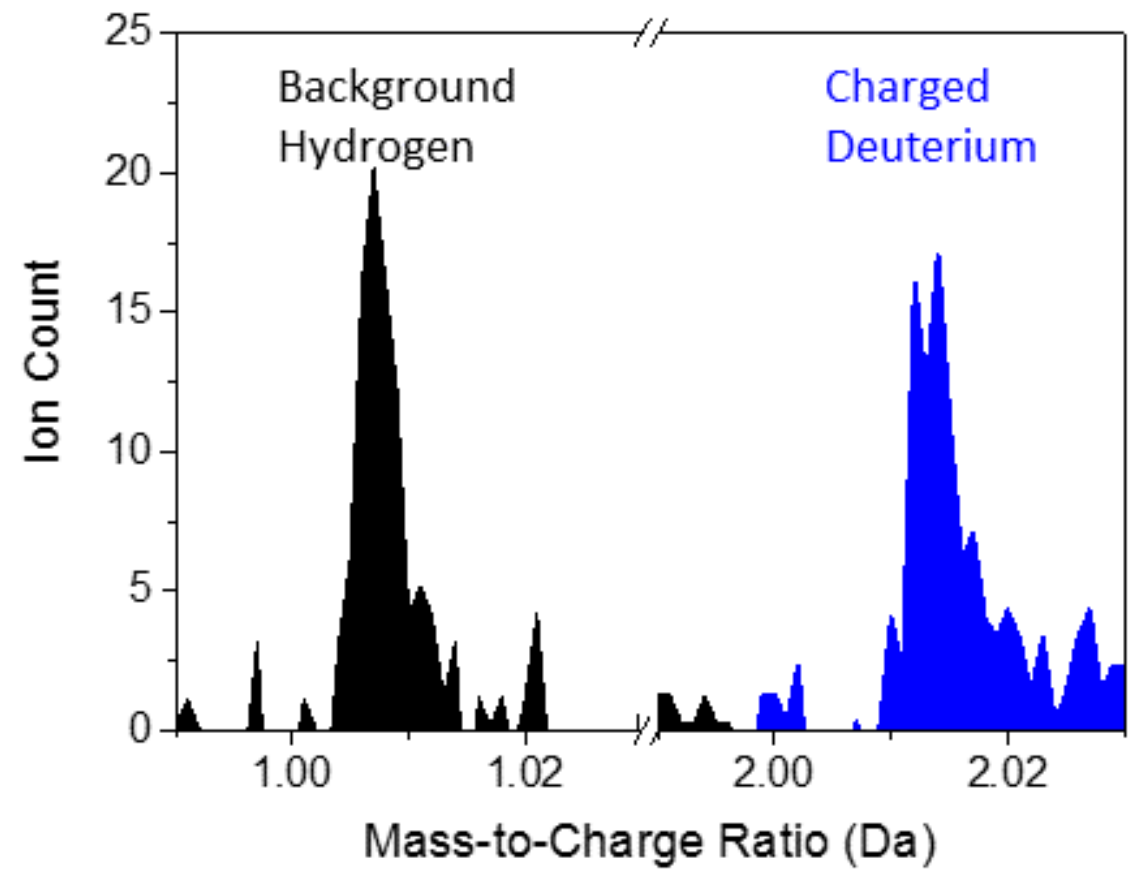

Figure 2. APT result of D-charged and cryo-transferred specimen. The mass spectrum shows strong D signal comparing to hydrogen reference. 